\title{
Large-scale emergent properties of an autocatalytic reaction-diffusion model subject to noise
}

\author{
David Hochberg, ${ }^{1, *}$ Felipe Lesmes, ${ }^{1, \dagger}$ Federico Morán, ${ }^{1,2, \hbar}$ and Juan Pérez-Mercader ${ }^{1, \S}$ \\ ${ }^{1}$ Centro de Astrobiología (CSIC-INTA), Carretera Ajalvir Km. 4, 28850 Torrejón de Ardoz, Madrid, Spain \\ ${ }^{2}$ Departamento de Bioquímica y Biología Molecular, Facultad de Ciencias Químicas, Universidad Complutense de Madrid, \\ Madrid, Spain
}

(Received 25 February 2003; published 24 December 2003)

\begin{abstract}
The nonequilibrium dynamic fluctuations of a stochastic version of the Gray-Scott (GS) model are studied analytically in leading order in perturbation theory by means of the dynamic renormalization group. There is an attracting stable fixed point at one-loop order, and the asymptotic scaling of the correlation functions is predicted for both spatial and temporally correlated noise sources. New effective three-body reaction terms, not present in the original GS model, are induced by the combined interplay of the fluctuations and nonlinearities.

DOI: 10.1103/PhysRevE.68.066114 PACS number(s): 02.50. $-\mathrm{r}, 05.10 . \mathrm{Cc}, 72.70 .+\mathrm{m}, 82.20 .-\mathrm{w}$
\end{abstract}

\section{INTRODUCTION}

The problem of pattern formation is a multidisciplinary challenge of great technological and scientific interest, and has been studied extensively in recent years by physicists, chemists, biologists, materials scientists, and others [1]. Much of the work to date has been devoted largely to general aspects of pattern-forming instabilities, pattern selection, waves and fronts, and direct numerical simulation of the idealized deterministic equations modeling the phenomena under study [2]. In this approach to modeling, it is reasonable to assume a continuum and coarse-grained description for the dynamic variables (degrees of freedom) and the model equations are frequently, though not always, of the reactiondiffusion type. Thus, one begins by writing down some specific deterministic model equations. This may be regarded as the phenomenological approach to the problem. However, a first principles derivation of the dynamical equations is obtained by removing the fast or short wavelength degrees of freedom from the microscopic description, and this process leads to unavoidable noise terms, representing the effects of internal fluctuations, which result from this small-scale elimination step [3]. Additionally, in the study of complex phenomena, there is typically no precise knowledge of many of the microscopic details, nor of the initial or boundary conditions needed to provide a complete description of the problem. The dynamics may also evolve in a medium (such as a background fluid) which provides external perturbations, environmental noise, and unpredictable disturbances. Nevertheless, one is interested in an explicit understanding of the system at long wavelengths. For all these reasons, it is natural to consider the influence of random noise on otherwise deterministic models and to study stochastic reactiondiffusion equations.

One of the simplest models of biochemical relevance leading to spatial and temporal patterns when diffusion is

\footnotetext{
*Electronic address: hochberg@laeff.esa.es

†Electronic address: lesmeszf@inta.es

*Electronic address: fmoran@solea.quim.ucm.es

${ }^{\S}$ Electronic address: mercader@laeff.esa.es;

http://www.cab.inta.es
}

included is that due to Gray and Scott [4]. Numerical simulations of deterministic systems have revealed a surprisingly large set of hitherto unknown complex and irregular patterns [5]. Because of the above considerations, and in regards to these recent findings, three questions that immediately come to mind are as follows: (a) How do the fluctuations affect the stability of an established pattern?, (b) What are the emergent properties, due to fluctuation effects, of such a system at long wavelengths? (c) How do the deterministic and stochastic effects compete? The purpose of this paper is to formulate carefully an analytic answer to the second question posed in (b). The latter will be carried out with the help of the dynamic renormalization group ( $R G)$. The questions raised in (a) and (c), of how noise influences pattern selection, will be investigated numerically and will be presented elsewhere. Striking numerical evidence for noise controlled pattern selfreplication is discussed in [6], where the replication rate is maximal for an optimal but small noise intensity.

We therefore consider a stochastic version of the GrayScott model [4], defined by the following system of stochastic partial differential equations:

$$
\begin{gathered}
\frac{\partial}{\partial t} V=\lambda U V^{2}-\mu V+D_{v} \nabla^{2} V+\eta_{v}(\mathbf{x}, t), \\
\frac{\partial}{\partial t} U=u_{0}-\lambda U V^{2}-\nu U+D_{u} \nabla^{2} U+\eta_{u}(\mathbf{x}, t) .
\end{gathered}
$$

In the absence of noise, (1) coincides with the Gray-Scott model [4], which is a variant of the autocatalytic Selkov model of glycolysis, corresponding to the following chemical reactions:

$$
\begin{gathered}
\stackrel{\lambda}{U}+2 V \rightarrow 3 V, \\
\stackrel{\mu}{\rightarrow} P, \\
{ }^{\nu} \\
U \rightarrow Q, \\
u_{0} \\
\rightarrow U .
\end{gathered}
$$


$V(\mathbf{x}, t)$ and $U(\mathbf{x}, t)$ represent the concentrations of the chemical species $U$ and $V$, and are functions of $d$-dimensional space $\mathbf{x}$ and time $t . \lambda$ is the reaction rate, $P$ and $Q$ are inert products, $\mu$ is the decay rate of $V$ and $\nu$ is the decay rate of $U$ and $u_{0}$ is the constant feed rate. In [5], the reaction rate parameter was simply set equal to unity: $\lambda=1$. Here we retain it as a free parameter, as we will see that it undergoes a nontrivial renormalization. A nonequilibrium constraint is represented by a feed term for $U$. The rate at which $U$ is supplied is positive if the concentration of $U$ drops below an equilibrium value and negative if it exceeds it. The equilibrium $U$ concentration is $u_{0} / \nu$, where $u_{0}$ is the feed rate constant. The chemical species $U$ and $V$ can diffuse with independent diffusion constants $D_{u}$ and $D_{v}$. All the model parameters are positive.

Any real chemical system is subjected to random fluctuations. We can include such effects in the GS model by means of noise terms that can, in principle, be additive or multiplicative. In this work we have chosen to investigate the influence of additive noise alone as a initial approach to the full stochastic problem in which both types of fluctuations can be simultaneously present. This is intended, therefore, as a first step towards incorporating fluctuation effects. This choice is influenced in part by the technical aspects of the dynamical renormalization group. From this perspective, additive noise, which enters the dynamics linearly, is technically easier to treat analytically in RG calculations. Accordingly, here we have opted to use independent additive colored noises, $\eta_{v}(\mathbf{x}, t)$ and $\eta_{u}(\mathbf{x}, t)$. First spatially correlated noise and then temporally correlated noise are considered. A few cautionary remarks regarding our incorporation of noise are in order. We emphasize that here we are adopting a simple phenomenological approach, wherein noise is added to the idealized deterministic equations. But, stochastic equations can also be derived from first principles. We refer to the methods that take a classical master equation, purporting to encode the precise microchemistry, to a continuum field theory [7-9]. This "second-quantized" formalism requires detailed knowledge of the microscopic master equation, and the end result of this method yields the stochastic PDE (SPDE) for the coarse-grained degrees of freedom and the (internal) noise correlations. A technical discrepancy comes up in applying phenomenological reaction-diffusion equations with real additive noise to pair-reaction kinetics (i.e., $V+V \rightarrow 0$ ), since in this particular case of particle annihilation, the SPDE derived from the fundamental microscopic master equation is complex and contains imaginary noise [10]. However, for standard Gribov processes (particle clustering reactions), the first-principles method does lead to a real SPDE with real additive noise. Thus, the ad hoc method is adequate for handling these cases. For external or environmental noise, the phenomenological strategy adopted here is also adequate since external fluctuations are typically specified only at the coarse-grained level.

The remainder of the paper is organized as follows. In Sec. II we specify the noise properties directly in Fourier space and derive the scaling laws that the model parameters in (1) obey when the stochastic equations are scale invariant (as they are in the neighborhood of any RG fixed point) and the general scaling form of the correlation functions of the composition fields is deduced in Sec. II A. This information will be needed when we apply the renormalization group in Sec. III. The fixed points and associated exponents are solved for and the RG flow is represented in a reduced twodimensional parameter space. The global flow patterns clearly illustrate the $d$ dependence of the flow, the role of the associated critical dimension, as well as the phenomena of crossover. In Sec. IV we discuss the emergence of effective three-body reaction terms not present in the original GS model that arise as a direct consequence of fluctuations and nonlinearity. Modifications and changes in the RG results due to the presence of temporally correlated noise are very briefly discussed in Sec. V. Conclusions and discussion are presented in Sec. VI.

\section{NAIVE SCALING PROPERTIES}

We can deduce the naive scaling laws that must hold if the stochastic equations (1) are to be form-invariant under a basic rescaling of both space and time. To do so, we specify the properties of the noise. In Fourier space, the noise correlations are given by

$$
\begin{aligned}
& \left\langle\eta_{v}(\mathbf{k}, \omega) \eta_{v}\left(\mathbf{k}^{\prime}, \omega^{\prime}\right)\right\rangle \\
& =2(2 \pi)^{d+1} A_{v} k^{-y_{v}} \delta^{d}\left(\mathbf{k}+\mathbf{k}^{\prime}\right) \delta\left(\omega+\omega^{\prime}\right), \\
& \left\langle\eta_{u}(\mathbf{k}, \omega) \eta_{u}\left(\mathbf{k}^{\prime}, \omega^{\prime}\right)\right\rangle \\
& =2(2 \pi)^{d+1} A_{u} k^{-y_{u}} \delta^{d}\left(\mathbf{k}+\mathbf{k}^{\prime}\right) \delta\left(\omega+\omega^{\prime}\right), \\
& \left\langle\eta_{v}(\mathbf{k}, \omega) \eta_{u}\left(\mathbf{k}^{\prime}, \omega^{\prime}\right)\right\rangle=0 .
\end{aligned}
$$

We assume all cumulants vanish, except the above, thus the noises are individually Gaussian, centered around zero and mutually uncorrelated. The independent noise amplitudes $A_{v}, A_{u}>0$ are positive definite and the noise exponents $y_{v}$ and $y_{u}$ are real free parameters.

Space, time, and the concentrations $U, V$, are rescaled by the following transformations:

$$
\begin{gathered}
\mathbf{x} \rightarrow s^{-1} \mathbf{x}, \\
t \rightarrow s^{-z} t, \\
V \rightarrow s^{-\chi_{v}} V, \\
U \rightarrow s^{-\chi_{u}} U .
\end{gathered}
$$

Here, $s>1$ is a convenient scale factor and $z$ is the so-called dynamic exponent. We initially allow for each concentration field to respond independently under the rescaling; this is reflected by the two a priori independent roughness exponents $\chi_{v}$ and $\chi_{u}$, respectively. However, the fact that the 
reaction rate $\lambda$ appears simultaneously in both the $U$ and $V$ equation requires that $\chi_{u}=\chi_{v} \equiv \chi$, as is easy to check. There is just a single roughness exponent to deal with.

Subjecting the equations (1) to this transformation, we find the model parameters scale naively (classically) in the following way:

$$
\begin{gathered}
\nu \rightarrow s^{z} \nu, \\
\mu \rightarrow s^{z} \mu, \\
D_{v} \rightarrow s^{z-2} D_{v}, \\
D_{u} \rightarrow s^{z-2} D_{u}, \\
u_{0} \rightarrow s^{z-\chi} u_{0}, \\
A_{v} \rightarrow s^{y_{v}-d+z-2 \chi} A_{v}, \\
A_{u} \rightarrow s^{y_{u}-d+z-2 \chi} A_{u}, \\
\lambda \rightarrow s^{2 \chi+z} \lambda_{u} .
\end{gathered}
$$

\section{Correlation functions}

Both the dynamic and roughness exponents govern the scaling form of the correlation functions of the concentration fields. To see this, consider the effect of a scale transformation on the $V$ field which we write more explicitly as

$$
V\left(s \mathbf{x}, s^{z} t\right)=s^{\chi} V(\mathbf{x}, t),
$$

which holds in the scaling regime (when the system is near one of its RG fixed points). Then the correlation function will scale as

$$
\langle V(\mathbf{x}, t) V(0,0)\rangle=s^{-2 \chi}\left\langle V\left(s \mathbf{x}, s^{z} t\right) V(0,0)\right\rangle=|\mathbf{x}|^{2} \chi \Phi\left(\frac{t}{|\mathbf{x}|^{z}}\right),
$$

where the scaling function $\Phi$ obeys the following asymptotic limits [11]:

$$
\lim _{u \rightarrow 0} \Phi(u)=\text { const, } \quad \lim _{u \rightarrow \infty} \Phi(u)=\text { const } \times u^{2} \chi / z .
$$

Thus, knowledge of both scaling exponents $(z, \chi)$ is required in order to predict the long-wavelength and long-time correlations of the fields. Similar considerations apply to the $\langle U U\rangle$ and cross-correlation $\langle V U\rangle$. In fact, the scaling of these latter two correlations will obey a relation similar to Eq. (7), except for a possibly different scaling function. Nevertheless, the limits in Eq. (8) are valid for all scaling functions, although the constants appearing there can be distinct. The tool best suited for the calculation of the required exponents is provided by the renormalization group, to which we next turn.

\section{RENORMALIZATION GROUP (RG) ANALYSIS}

Since we are interested in the emergent scaling properties of the model, we focus on the hydrodynamic limit, that is, the long-time $t \rightarrow \infty$ and long-distance $\mathbf{x} \rightarrow \infty$ limits. The dynamical $\mathrm{RG}[12,13]$ is a powerful tool for computing and analyzing the asymptotic properties of out-of-equilibrium stochastic systems.

In order to calculate the scaling exponents in the asymptotic limit, the linear and nonlinear parts of the equations of motion can be reorganized so as to allow a perturbative calculation in powers of the nonlinear parameter $\lambda$. After Fourier transforming, the coupled stochastic equations of motion (1) can be rewritten as

$$
\begin{aligned}
V(\mathbf{k}, \omega)= & G_{v 0}(\mathbf{k}, \omega) \eta_{v}(\mathbf{k}, \omega)+G_{v 0}(\mathbf{k}, \omega) \lambda \\
& \times \int \frac{d^{d} \mathbf{k}_{\mathbf{1}}}{(2 \pi)^{d}} \frac{d \omega_{1}}{2 \pi} \frac{d^{d} \mathbf{k}_{\mathbf{2}}}{(2 \pi)^{d}} \frac{d \omega_{2}}{2 \pi} V\left(\mathbf{k}_{\mathbf{1}}, \omega_{1}\right) V\left(\mathbf{k}_{\mathbf{2}}, \omega_{2}\right) \\
& \times U\left(\mathbf{k}-\mathbf{k}_{\mathbf{1}}-\mathbf{k}_{\mathbf{2}}, \omega-\omega_{1}-\omega_{2}\right), \\
U(\mathbf{k}, \omega)= & G_{u 0}(\mathbf{k}, \omega) \eta_{u}(\mathbf{k}, \omega)+G_{u 0}(\mathbf{k}, \omega) \\
& \times(2 \pi)^{d+1} \delta^{d}(\mathbf{k}) \delta(\omega) u_{0}-G_{u 0}(\mathbf{k}, \omega) \lambda \\
& \times \int \frac{d^{d} \mathbf{k}_{\mathbf{1}}}{(2 \pi)^{d}} \frac{d \omega_{1}}{2 \pi} \frac{d^{d} \mathbf{k}_{\mathbf{2}}}{(2 \pi)^{d}} \frac{d \omega_{2}}{2 \pi} V\left(\mathbf{\mathbf { k } _ { \mathbf { 1 } }}, \omega_{1}\right) V\left(\mathbf{k}_{\mathbf{2}}, \omega_{2}\right) \\
& \times U\left(\mathbf{k}-\mathbf{k}_{\mathbf{1}}-\mathbf{k}_{\mathbf{2}}, \omega-\omega_{1}-\omega_{2}\right),
\end{aligned}
$$

where the bare propagators (or, response functions) $G_{v 0}$ and $G_{u 0}$ are defined by

$$
G_{v 0}(\mathbf{k}, \omega)=\frac{1}{\mu+D_{v} k^{2}-i \omega},
$$

$$
G_{u 0}(\mathbf{k}, \omega)=\frac{1}{\nu+D_{u} k^{2}-i \omega} .
$$

The wave-number modulus is denoted by $k=|\mathbf{k}|$. Define the effective propagators by

$$
\begin{gathered}
V(\mathbf{k}, \omega) \equiv G_{v}(\mathbf{k}, \omega) \eta_{v}(\mathbf{k}, \omega) \\
U(\mathbf{k}, \omega) \equiv G_{u}(\mathbf{k}, \omega)\left\{\eta_{u}(\mathbf{k}, \omega)+(2 \pi)^{d+1} \delta^{d}(\mathbf{k}) \delta(\omega) u_{0}\right\}
\end{gathered}
$$

Substituting these into the set of integral equations (9) yields a set of equations that can be solved iteratively to any order in $\lambda$. To this end, it is best to handle the expansion via diagrams, and the above equation (9) are represented diagrammatically as follows: 


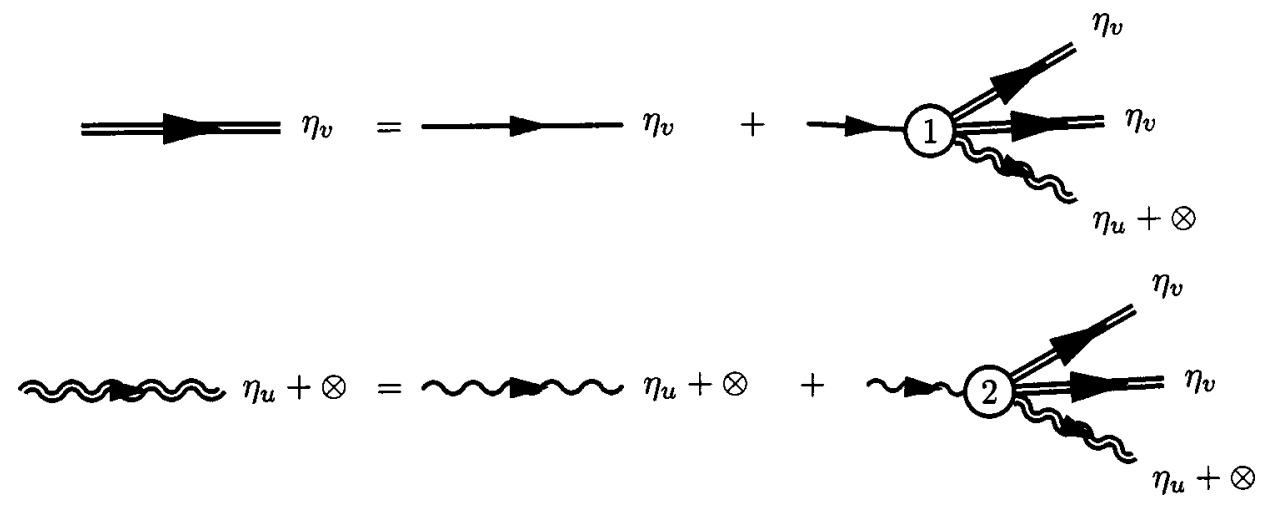

The directed double straight (wiggly) line symbol represents the effective $V$ propagator ( $U$ propagator) defined above: straight and wiggly lines correspond to the $V$ sector, and $U$ sector, respectively. The single directed lines stand for the bare propagators (10). It is understood that each directed line carries wave number $\mathbf{k}$ and frequency $\omega$, which is conserved at all vertices, as can be seen from Eq. (9). We do not write down this dependence explicitly on the graphs in order to avoid clutter. The nonlinear coupling terms, or vertices, are represented by the directed four-pronged symbols, of which there are two, and these are denoted by the encircled numer- als (1) and (2), respectively. The zero mode $u_{0}$ in the second equation in (9) is indicated above by a $\otimes$. The perturbation expansion can now be performed efficiently without having to carry along lengthy and tedious algebraic expressions. For further details on using graphical methods to solve stochastic differential equations, see the Appendixes of [14]. The calculation of the propagator then follows from a graphical iteration of these expressions, amputating one noise factor from the legs, followed by an averaging over the remaining noise factors. The results at one-loop level are indicated as follows:

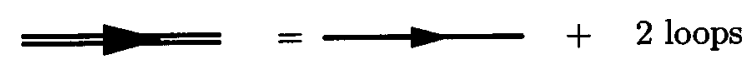

๙ $\approx$ ก $=\sim \sim \sim$

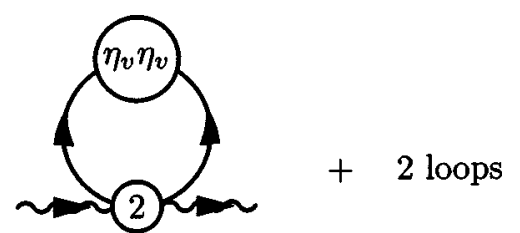

Note that only the $U$ propagator receives corrections at one loop, whereas the $V$ propagator is unchanged to this order. Furthermore, the $v$ noise is what "drives" the renormalization of the $U$ propagator. The loop expansion for the vertices is indicated below. One takes the vertices and iterates, to a desired order in the coupling $\lambda$, by replacing directed double lines using the above equations of motion. Then, three noise factors (two $\eta_{v}$ 's and one $\eta_{u}$ ) are amputated and the resultant graphs are averaged over the remaining noises. A combinatorial factor of 4 appears after counting all possible noise contractions. We thus obtain
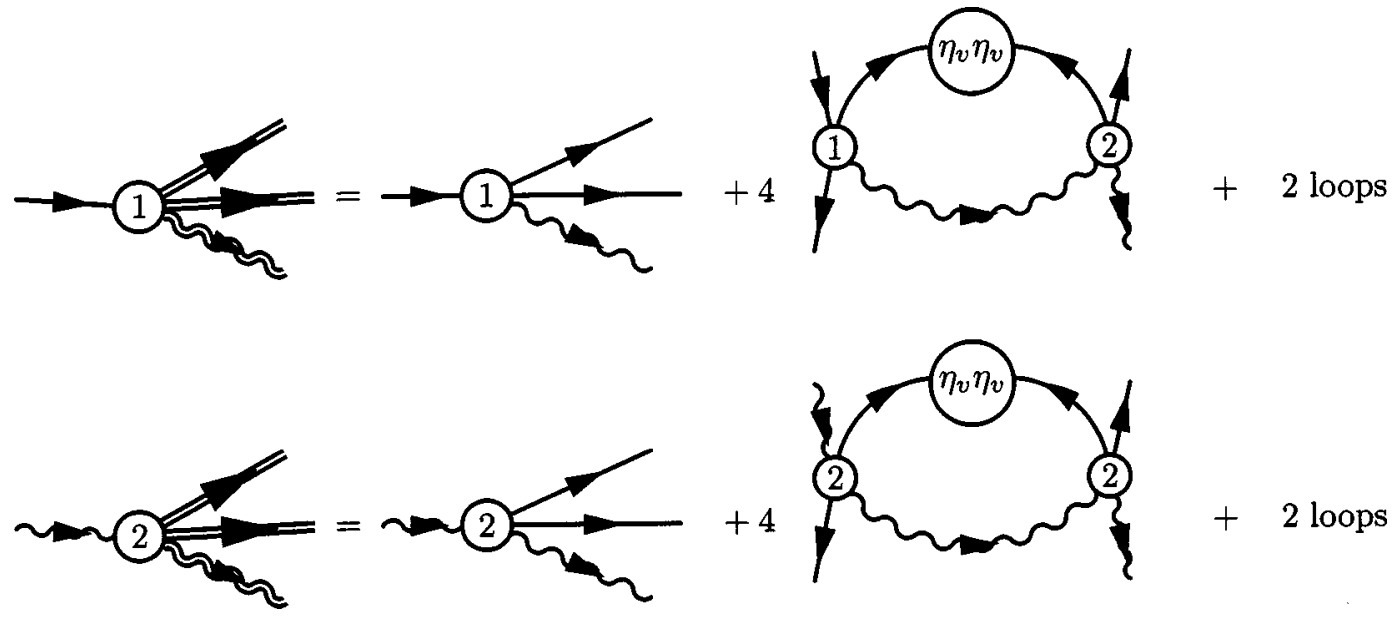
The noise spectral functions receive no corrections at oneloop order. We point out that although $\lambda$ is employed as a formal expansion parameter, the bonafide and dimensionless perturbation parameter $g$ [see Eq. (14)] is a combination of this, a noise amplitude, a decay constant, a diffusion constant, and a short distance (or ultraviolet) cutoff needed for convergence of the integrals. Moreover, we expand in loops rather than in powers of $g$. Physically, the loop expansion is an expansion in powers of the noise amplitude [15]. We note that at leading order in loops, the perturbation expansion indicates that only the $U$ field propagator and the nonlinear coupling $\lambda$ receive corrections. The noise amplitudes and the $V$ propagator are unchanged at one-loop order. At two-loop order, all propagators, the noise amplitudes, as well as the vertices, do receive corrections. However, there are some important issues at leading order that need to be understood.

The dynamical RG transformation is carried out in two steps [13].

(1) High momenta components, in the momentum shell $\Lambda e^{-\delta l}<|\mathbf{k}|<\Lambda$, at and below the cutoff $\Lambda$, are integrated out. Note: $s=e^{l}$.

(2) A change of scale restores the cutoff to the value $\Lambda$.

Further details of the calculation are given in Appendixes $\mathrm{A}$ and $\mathrm{B}$. Working with infinitesimal parameter $\delta l$ gives rise to differential equations governing the RG flow in parameter space,

$$
\begin{gathered}
\frac{d \nu}{d l}=z \nu+\frac{\lambda A_{v} K_{d} \Lambda^{d-y_{v}}}{\left(\mu+D_{v} \Lambda^{2}\right)}, \\
\frac{d \mu}{d l}=z \mu, \\
\frac{d D_{v}}{d l}=(z-2) D_{v}, \\
\frac{d D_{u}}{d l}=(z-2) D_{u}, \\
\frac{d u_{0}}{d l}=(z-\chi) u_{0}, \\
\frac{d A_{v}}{d l}=\left(y_{v}-d+z-2 \chi\right) A_{v}, \\
\frac{d A_{u}}{d l}=\left(y_{u}-d+z-2 \chi\right) A_{u}, \\
\frac{d \lambda}{d l}=\left[2 \chi+z-\frac{D_{v} K_{d} \Lambda^{d-y_{v}}}{\left(\mu+D_{v} \Lambda^{2}\right)\left(\mu+\nu+D_{v} \Lambda^{2}+D_{u} \Lambda^{2}\right)}\right] \lambda .
\end{gathered}
$$

$K_{d}=S_{d} /(2 \pi)^{d}$ where $S_{d}$ is the surface area of a $d$-dimensional sphere. As already noted in the corresponding graphs, at one-loop order, only two out of the total of eight model parameters run with scale, namely, the decay rate $\nu$, of the $U$ field and the nonlinear coupling $\lambda$. Note, moreover, that at this order, the corrections are driven by the $v$ noise, but not the $u$ noise. Despite the relative simplicity of these equations, nontrivial RG fixed points and flow already result at this leading order perturbation.

\section{A. Fixed points and dynamic scaling}

We determine the fixed points and associated exponents implied by the above equations (13). We can analyze the RG flow in a reduced two-dimensional parameter space by introducing the pair of dimensionless couplings defined by

$$
\begin{gathered}
g=\lambda A_{v} K_{d} \frac{\Lambda^{d-y_{v}-2}}{\nu D_{v}}, \\
h=\left(D_{v}+D_{u}\right) \frac{\Lambda^{2}}{\nu},
\end{gathered}
$$

for then the RG equations (13) can be written as

$$
\begin{gathered}
\dot{\nu}=\nu(z+g), \\
\dot{\lambda}=\lambda\left(2 \chi+z-\frac{4 g}{1+h}\right) .
\end{gathered}
$$

We do not write out the remaining trivial RG equations. Note we have taken $\mu=0$ from the outset. This choice is necessary in order to obtain a nontrivial $g \neq 0$ fixed point solution of Eq. (13). Here the overdot stands for the derivative $d / d l$. In terms of $g$ and $h$, the nontrivial part of the RG flow is governed by the pair of equations

$$
\begin{gathered}
\dot{g}=g\left(y_{v}-d+2-g-\frac{4 g}{1+h}\right), \\
\dot{h}=-(2+g) h .
\end{gathered}
$$

The fixed points can be solved for by looking for all zeroes of the pair (18), (19), to be denoted as $\left(g^{*}, h^{*}\right)$ while the associated fixed point exponents are obtained by substituting the solutions of $\dot{g}=0$ and $\dot{h}=0$ into (16), (17); solving for the zeroes of this latter pair then yields the exponents, denoted by $\left(z^{*}, \chi^{*}\right)$.

We first search for all nontrivial fixed points. These correspond to $g * \neq 0$, since $g$ is proportional to the nonlinear coupling $\lambda$. There are two cases to be distinguished.

Case (a). $g^{*}=-2$ and $1+h^{*}=8 /\left(d-y_{v}-4\right)$. The associated exponents are $z^{*}=2$ and $\chi^{*}=\left(2-d+y_{v}\right) / 2$. Note that the combination $\left(z^{*}-2 \chi^{*}-d+y_{v}\right) \equiv 0$ vanishes identically. Thus, if we choose $u_{0}=0$, and $y_{u}=y_{v}$, all remaining RG equations (13) are stationary. Note for this fixed point solution, the stochastic GS model is in the same universality class as the linear Edwards-Wilkinson model [14].

Case (b). $h^{*}=0$ and $g^{*}=\left(y_{v}-d+2\right) / 5$. The exponents associated with this fixed point are $z^{*}=-\left(y_{v}-d+2\right) / 5$ and $\chi^{*}=\left(y_{v}-d+2\right) / 2$. Again we choose $u_{0}=0$, then all remaining RG equations are automatically stationary except that the noise amplitudes decay to zero on approaching this fixed 


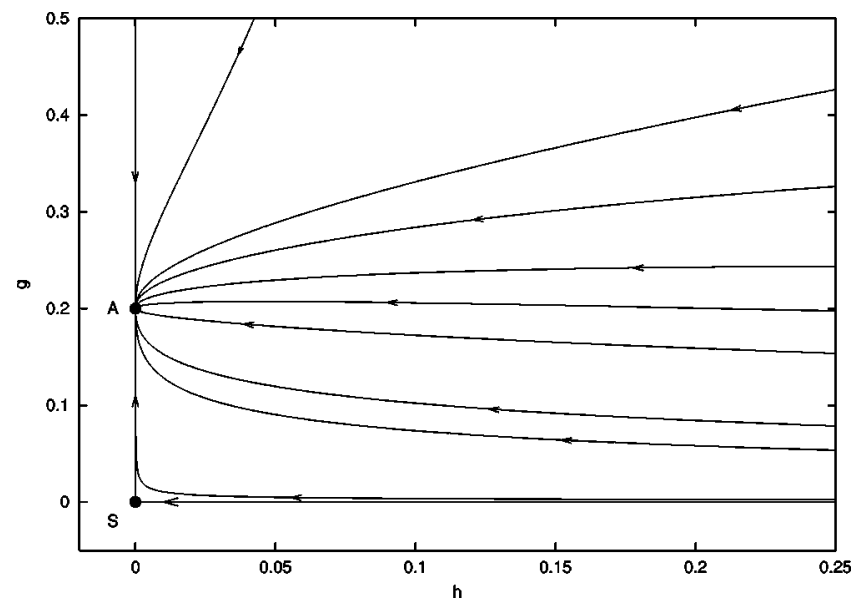

FIG. 1. A projection of the RG flow in Eqs. (18) and (19). Two fixed points: $A$ is attractive, $S$ is a saddle point. This is plotted for $\epsilon>0$.

point: $A_{v}(l)=A_{v} e^{l\left(z-2 \chi-d+y_{v}\right)} \rightarrow 0$ as $l \rightarrow \infty$ and similarly for $A_{u}(l)$. This limit holds provided that $d<12+y_{v}$, and $d$ $<12+y_{u}$ for the limit of $A_{u}$.

Since the model (1) is defined as having all non-negative parameters, we must exclude the solution in case (a) as being physically spurious. This leaves us with the nontrivial fixed point of case (b), which is an attractive fixed point and is indicated by the symbol $A$ in the flow graph; refer to Fig. 1.

There is one trivial fixed point: this is a saddle point, as indicated by $S$ in the flow graph Fig. 1. This corresponds to $g^{*}=0$ and $h^{*}=0$. The exponents are found to be $z^{*}=0$ and $\chi^{*}=\left(y_{v}-d\right) / 2$. This is a fixed point solution to all equations in (13) provided $y_{v}=y_{u}$ and again upon setting $u_{0}=0$.

\section{B. RG flow}

The RG flow in the regions of parameter space surrounding the fixed points is obtained by numerically integrating the differential equations (18), (19) for various choices of distinct initial conditions chosen from within the basins of attraction or repulsion of the fixed points. The results are shown in Figs. 1, 2, and 3. The topology, the direction of the flow, and the fixed point stability are controlled by the single parameter epsilon $\epsilon=2-d+y_{v}$. In deriving cases (a) and (b) above, we tacitly assumed that $\epsilon>0$. The corresponding flow is plotted in Fig. 1. The origin is a saddle point and there is one asymptotically stable fixed point, as shown there. For very small initial $g$, the effective dynamics will flow towards $S$ and then be repelled to $A$. Thus, the system exhibits crossover. In the vicinity of $S$, the correlations of the concentrations therefore scale as $(r=|\mathbf{x}|)$

$$
\langle V(\mathbf{x}, t) V(0,0)\rangle \sim r^{\left(y_{v}-d\right)},
$$

whereas on approaching the point $A$, they scale according to

$$
\begin{aligned}
\langle V(\mathbf{x}, t) V(0,0)\rangle & \sim r^{\left(y_{v}-d+2\right)} \quad \text { for } r \rightarrow \infty \\
& \sim t^{-5} \text { for } t \rightarrow \infty .
\end{aligned}
$$

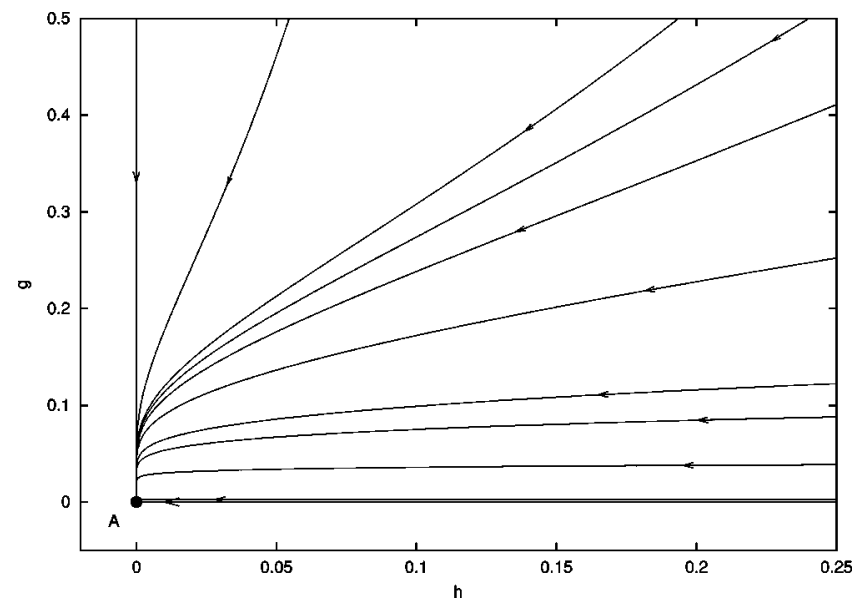

FIG. 2. A projection of the RG flow in Eqs. (18) and (19). One fixed point: $A$ is attractive. This is plotted for $\epsilon=0$.

If $\epsilon=0$, there is no fixed point at nonzero $g$. The origin $(0,0)$ changes from a saddle point to an attracting sink as $\epsilon \rightarrow 0$. The critical dimension is defined by $\epsilon=0$, and is given by $d_{c}=2+y_{v}$. This is the dimension below which fluctuations are relevant. For example, for white noise, $d_{c}=2$, which is also the critical dimension of the EdwardsWilkinson model [14].

For $\epsilon<0$, there is only the trivial fixed point $\mathbf{A}$ at the origin. Both these cases are shown in Figs. 2 and 3, respectively. In the neighborhood of this point, the correlations again scale as in Eq. (20).

\section{INDUCED THREE-BODY REACTIONS}

Consider the graphical representation of the stochastic equations of motion written in Eq. (9) and which are displayed below Eq. (12). In arriving at the perturbative expansion for the vertex representing the chemical reaction $U V^{2}$ in Eq. (1), the steps used in the graphical method involved replacing two of the vertex legs with the exact expression for $V(\mathbf{k}, \omega)$ and the third remaining leg with the exact expression for $U(\mathbf{k}, \omega)$ using Eq. (9). This step must be iterated

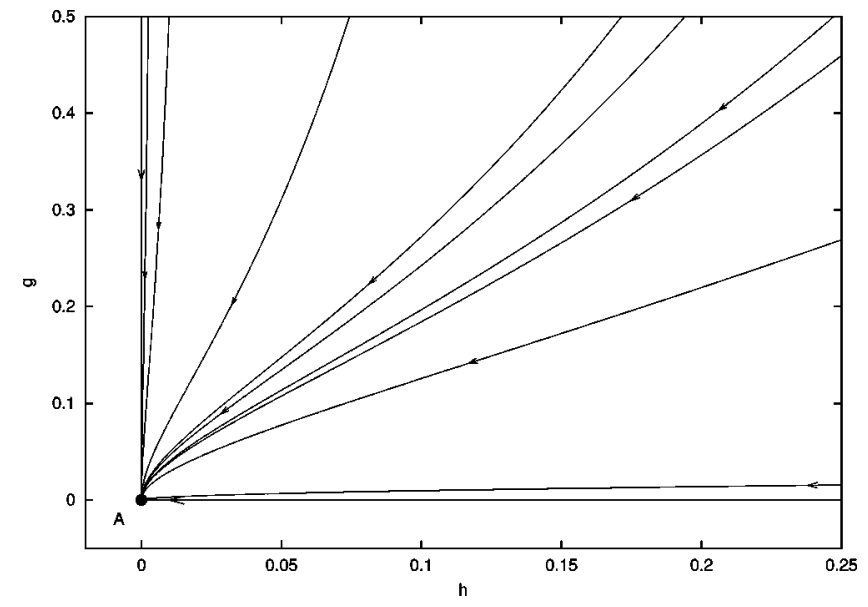

FIG. 3. A projection of the RG flow in Eqs. (18) and (19). One fixed point: $A$ is attractive. This is plotted for $\epsilon<0$. 
once more in order to obtain all one-loop diagrams. At this intermediate stage, however, we generate a set of tree diagrams (i.e., containing no loops) with all legs terminating either in an $\eta_{v}$ or in an $\eta_{u}$ noise factor. Now, the effective $U V^{2}$ vertex results after amputating (i.e., removing) two $\eta_{v}$ 's and one $\eta_{u}$ noise factor from each tree diagram and then averaging over the remaining noise factors. The averaging step makes use of the noise correlation functions specified in Eq. (3). The combinatorial factor for each diagram is found by counting all possible noise contractions that give rise to it. In this way, we arrived at the one-loop expansion for $\lambda$ depicted diagramatically above in Sec. III.

However, at the step leading to the set of tree diagrams, one can instead amputate two $\eta_{u}$ 's and one $\eta_{v}$ noise from the tree diagrams and then average over the remaining noises or, alternatively, amputate three $\eta_{v}$ factors and then average. The sequence of noise factor amputations can lead to different allowed reaction kinetics. Thus, at the one-loop level, in addition to the Gray-Scott $U V^{2}$ reaction, one also encounters the following (induced) effective reactions involving a triple product of chemical concentrations:

$$
U^{2} V \text { or } V^{3} \text {. }
$$

It is important to realize that these are alternative but exclusive reactions, in that noise-factor amputation can produce one reaction, say $\left(U^{2} V\right)$, or the other $\left(V^{3}\right)$, but not both simultaneously. This is simply because each alternative reaction derives from the same tree graph, the only difference is due to the sequence of noise amputations and subsequent averaging steps. Note it is not possible to amputate three $\eta_{u}$ factors at one loop level (this would have led to a $U^{3}$ reaction, which is therefore ruled out from the effective induced dynamics at this order). Both reactions in Eq. (23) are proportional to $\lambda^{2}$ at one-loop order. Moreover, since at one loop both concentration fields $U$ and $V$ scale with the same exponent $\chi$, these effective three-body reactions scale as $s^{3 \chi}$ and will be relevant (or, irrelevant) if and only if the original GS reaction is. Most importantly, the new reactions do not destroy the scale invariance of Eq. (1) at the fixed points.

Physically, this means that at one-loop order in the fluctuations, corrections are not only induced in the original GS reaction, leading to its renormalization [i.e., the last equation in Eq. (13)], but that a new effective reaction is generated. This new reaction is not present in the GS model (i.e., does not exist at tree level) but is a direct consequence of the combined effect of noise and nonlinear terms in Eq. (1). In this sense, the stochastic version of the GS is not renormalizable at long wavelengths because new relevant reaction terms are thereby induced. The large scale chemistry that emerges at the attractive fixed point $A$ is GS with corresponding effective (renormalized) parameters plus one of the alternative induced reactions written in (24) or in (25). This is interesting because it indicates that at large scales, the coarse-grained theory (1) represents an apparently distinct chemistry.

In terms of chemical reactions, the new reaction vertices would correspond to production of $V$ and or $U$ molecules in one of the alternative pathways indicated below. In the case of the vertex $U^{2} V$, we have various pathways

$$
V+2 U \rightarrow\left\{\begin{array}{c}
3 U \\
2 V+U \\
3 V,
\end{array}\right.
$$

whereas for the case of the vertex $V^{3}$,

$$
3 V \rightarrow\left\{\begin{array}{c}
3 U \\
2 V+U \\
V+2 U .
\end{array}\right.
$$

Thus, for the case of the induced $U^{2} V$ vertex, and for the specific pathway $V+2 U \rightarrow 2 V+U$, the effective one-loop reaction dynamics at the attractive fixed point is given by the following deterministic equations:

$$
\begin{gathered}
\frac{\partial}{\partial t} V=\lambda^{*} U V^{2}+\left(\lambda^{*}\right)^{2} U^{2} V+D_{v} \nabla^{2} V, \\
\frac{\partial}{\partial t} U=-\lambda^{*} U V^{2}-\left(\lambda^{*}\right)^{2} U^{2} V-\nu^{*} U+D_{u} \nabla^{2} U,
\end{gathered}
$$

where $\lambda^{*}$ and $\nu^{*}$ denote the fixed-point values of $\lambda$ and $\nu$, respectively. Recall that only these two parameters renormalize nontrivially at one-loop order. Moreover, from the fixed point analysis case (b) given in Sec. III A, we recall the solution $A$ is obtained for $\mu=0, u_{0}=0$ and both the noise amplitudes decay to zero upon approaching the fixed point provided $d<12+y$. For an attracting fixed point, $\epsilon=2-d$ $+y>0$ which requires $d<2+y$, which is the stronger inequality. Alternatively, for the case of the one-loop induced three-body reaction $V^{3}$, and for the pathway $3 V \rightarrow 2 V+U$, the effective one-loop reaction dynamics at the attractive fixed point is given by the pair of deterministic equations:

$$
\begin{gathered}
\frac{\partial}{\partial t} V=\lambda * U V^{2}-(\lambda *)^{2} V^{3}+D_{v} \nabla^{2} V, \\
\frac{\partial}{\partial t} U=-\lambda * U V^{2}+(\lambda *)^{2} V^{3}-\nu^{*} U+D_{u} \nabla^{2} U .
\end{gathered}
$$

The effective reaction dynamics derived above results from the lowest order one-loop perturbative RG calculations. If the RG program is carried out to higher order (for example, to two-loop order), then these one-loop induced terms would have to be taken into account.

\section{TEMPORALLY CORRELATED NOISE}

Starting from a microscopic description, the elimination of the fast degrees of freedom leading to (1) can in principle result in noise with long-range correlations in both space and time. It is therefore of interest to investigate the influence of temporally correlated noise on the present phenomenological model. It is straightforward to extend the RG analysis to incorporate long range temporal correlations. Since the basic calculational steps are similar to those employed above, we 
will be concise and list only the salient features specific to temporal correlations.

So, in place of (3), we consider Gaussian noise with correlations that behave asymptotically as follows:

$$
\begin{aligned}
\left\langle\eta_{v}(\mathbf{k}, \omega) \eta_{v}\left(\mathbf{k}^{\prime}, \omega^{\prime}\right)\right\rangle= & 2(2 \pi)^{d+1} A_{v} k^{-y_{v}} \omega^{-2 \theta_{v}} \delta^{d}\left(\mathbf{k}+\mathbf{k}^{\prime}\right) \\
& \times \delta\left(\omega+\omega^{\prime}\right), \\
\left\langle\eta_{u}(\mathbf{k}, \omega) \eta_{u}\left(\mathbf{k}^{\prime}, \omega^{\prime}\right)\right\rangle= & 2(2 \pi)^{d+1} A_{u} k^{-y_{u}} \omega^{-2 \theta_{u}} \delta^{d}\left(\mathbf{k}+\mathbf{k}^{\prime}\right) \\
& \times \delta\left(\omega+\omega^{\prime}\right),
\end{aligned}
$$

where the exponents $\theta_{v}, \theta_{u}$ control the range of the temporal correlations; the limit of purely spatial correlations is recovered by setting $\theta_{v}=\theta_{u}=0$.

Scaling properties of the noise amplitudes are modified as follows:

$$
\begin{aligned}
& A_{v} \rightarrow s^{y_{v}-d-2 \chi+z\left(1+2 \theta_{v}\right)} A_{v}, \\
& A_{u} \rightarrow s^{y_{u}-d-2 \chi+z\left(1+2 \theta_{u}\right)} A_{u},
\end{aligned}
$$

while the remainder of the relations in (5) are unchanged. The steps needed to carry out the perturbation expansion and associated diagrammatic development of (1) are the same as before; only now, the noise factors appearing there and in the diagrams are those corresponding to Eqs. (28). The calculation of the required loop diagrams and integrals follows the same basic steps as outlined in Appendixes A and B. Due to (28) the integration over internal loop frequency $\Omega$ is much more complicated, though still analytically tractable [16]. The two nontrivial one-loop differential RG equations in (13) are modified accordingly:

$$
\begin{gathered}
\frac{d \nu}{d l}=z \nu+\csc \left[\left(1+2 \theta_{v}\right) \frac{\pi}{2}\right] \frac{\lambda A_{v} K_{d} \Lambda^{d-y_{v}}}{\left(\mu+D_{v} \Lambda^{2}\right)^{1+2 \theta_{v}}} \\
\frac{d \lambda}{d l}=\left(2 \chi+z-4 \csc \left[\left(1-2 \theta_{v}\right) \frac{\pi}{2}\right] \lambda A_{v} K_{d} \Lambda^{d-y_{v}}\left(\nu+D_{u} \Lambda^{2}\right)\right. \\
\left.\times \frac{\left(\nu+D_{u} \Lambda^{2}\right)^{-1-2 \theta_{v}}-\left(\mu+D_{v} \Lambda^{2}\right)^{-1-2 \theta_{v}}}{\left(\mu+D_{v} \Lambda^{2}\right)^{2}-\left(\nu+D_{u} \Lambda^{2}\right)^{2}}\right) \lambda
\end{gathered}
$$

and the allowed range of the noise exponent is $-\frac{1}{2}<\theta_{v}$ $<\frac{1}{2}$. As in the case of pure spatially correlated noise, we can analyze the RG flow and fixed points in terms of a convenient choice of dimensionless parameters (taking $\mu=0$ from the outset):

$$
\begin{gathered}
g=\lambda A_{v} K_{d} \csc \left[\left(1+2 \theta_{v}\right) \frac{\pi}{2}\right] \frac{\Lambda^{d-y_{v}}}{\nu\left(D_{v} \Lambda^{2}\right)^{1+2 \theta_{v}}}, \\
h_{1}=D_{v} \frac{\Lambda^{2}}{\nu} \\
h_{2}=D_{u} \frac{\Lambda^{2}}{\nu} .
\end{gathered}
$$

This should be contrasted with the pair used in Sec. III A: here the temporal correlations do not allow one to combine $h_{1}$ and $h_{2}$ consistently into a single unified variable $\left(h_{1}\right.$ $\left.+h_{2}\right) \rightarrow h$, as we did above.

In terms of these variables, the RG equations can be written as

$$
\begin{gathered}
\dot{g}=g\left(y_{v}-d+2\left(1+2 \theta_{v}\right)-g-4 g\left(1+h_{2}\right)\right. \\
\left.\times \frac{\left(\frac{h_{1}}{1+h_{2}}\right)^{1+2 \theta_{v}}-1}{\left(1+h_{1}+h_{2}\right)\left(-1-h_{2}+h_{1}\right)}\right), \\
\dot{h}_{1}=-h_{1}(2+g), \\
\dot{h}_{2}=-h_{2}(2+g) .
\end{gathered}
$$

The fixed points are solved for by looking for all the zeroes of the triplet of equations in (33). As before, there are two nontrivial fixed points and one trivial fixed point. One of the nontrivial fixed points corresponds to $g^{*}=-2, h_{1}^{*}$ and $h_{2}^{*}$ are arbitrary constants, and we discard it for the reasons given earlier [see comments concerning case (a) in Sec. III A $]$. The other nontrivial point corresponds to $g^{*}=\left[y_{v}\right.$ $\left.-d+2\left(1+2 \theta_{v}\right)\right] / 5$ and $h_{1}^{*}=h_{2}^{*}=0$. The associated exponents are found to be $z^{*}=-\left[y_{v}-d+2\left(1+2 \theta_{v}\right)\right] / 5$ and $\chi^{*}=\left[y_{v}-d+2\left(1+2 \theta_{v}\right)\right] / 2$. Previous results pertaining to pure spatially correlated noise are immediately recovered by setting $\theta_{v}=0$ in these expressions. The trivial fixed point corresponds to $g^{*}=h_{1}^{*}=h_{2}^{*}=0$, with exponents $z^{*}=0$ and $\chi^{*}=\left(y_{v}-d\right) / 2$, results which are seen to be identical to those of the purely spatially correlated case. In this case, the flow is governed by the parameter $\epsilon=y_{v}-d+2\left(1+2 \theta_{v}\right)$. The RG flow is qualitatively the same as before, so in this respect, temporally correlated noise leads to no new features at one-loop order.

\section{CONCLUSIONS AND DISCUSSION}

We have studied the large wavelength and long time limits of the Gray-Scott model subject to random fluctuations. We carried this out for additive noise containing long range correlations in space and in time, which leads one to consider a set of coupled stochastic partial differential equations. The noise is intended to model in part the combination of coarsegrained external fluctuations, environmental noise, and also imprecise knowledge of initial and boundary conditions. The asymptotic behavior of this system is revealed from applying the dynamical renormalization group combined with perturbation theory. This behavior is summarized by flows in parameter space, which indicate how the parameters of the dynamic model change under coarse graining; general points in this space represent the dynamics effective at the corresponding space and time scale. The fixed points control the effective dynamics at the largest scales and corresponding correlation functions (in terms of the chemical concentrations or 
composition fields) are power laws depending on a pair of scaling exponents. It will be noted that the information obtained here is of a statistical nature, since here one deals in correlation functions, which are themselves probabilities. These correlations provide a measure of the long wavelength properties of the patterns that can form in the presence of fluctuations.

The RG calculation was carried out to one-loop order. At this lowest order, only two out of eight of the model parameters receive corrections, yet there is already a nontrivial infrared stable fixed point and the phenomena of crossover. We have also identified the critical dimension below which fluctuations are relevant. This dimension is $d_{c}=2+y_{v}$ for spatially correlated noise or $d_{c}=2+y_{v}+2\left(1+2 \theta_{v}\right)$, for noise with both spatial and temporal correlations, respectively. In the limit of large scales (after coarse graining) the properties of the system converge towards a simple solution with fractal properties (i.e., exhibiting scale invariance) as shown in Fig. (1). The existence of this solution depends on the sign of $\epsilon$. The nontrivial scale invariant solution is present only for positive values of $\epsilon$, when the fluctuations become important and then emergent behavior arises. Moreover, we find that the combined interplay of the nonlinearity and the fluctuations lead to effective three-body reaction terms not present in the original GS model. At one-loop order in the fluctuations the new reaction vertices correspond to three-body molecular reactions with various alternative pathways as expressed in Eqs. (24) and (25).

The renormalized reaction-diffusion equations therefore can be represented by a chemistry apparently distinct from the original GS reaction. For small noise amplitudes, a oneloop calculation should be adequate to capture some of the salient features of stochastic reaction-diffusion dynamics. In fact, simple white noise can lead to rather striking effects. Preliminary numerical calculations demonstrate clearly that both pattern selection and dynamic pattern replication can be controlled by adding white noise to the deterministic GS model [6]. These studies raise important questions pertaining to the role of noise in both chemical and biological selforganization and the environmental selection of emergent properties.

At one-loop order, temporally correlated noise leads to no noteworthy features in the RG analysis, though that conclusion may change at next higher order. Temporally correlated fluctuations can lead to complex scaling exponents which signal the presence of hierarchical structures [17]. Such structures are absent at one-loop order.

\section{ACKNOWLEDGMENTS}

We thank Marcel Vlad for reading a preliminary version of this paper and for providing us with useful comments. The research of F.M. is supported in part by the Grant No. BMC2000-0764 from MCyT (Spain). F.L. acknowledges financial support provided by INTA for training in astrobiology. D.H. and J.P-M. are supported by funds from INTA, Comunidad Autónoma de Madrid, and Grant No. BXX20001385 from MCyT (Spain).

\section{APPENDIX A: PROPAGATOR RENORMALIZATION}

Details of the propagator renormalization are give here. As the diagrams for the expanded propagators show, at one loop only the $U$ propagator receives corrections. Reverting back from diagrams to corresponding algebraic quantities, we have that

$$
\begin{aligned}
G_{u}(\mathbf{k}, \omega)= & G_{u 0}(\mathbf{k}, \omega)+\lambda\left[G_{u 0}(\mathbf{k}, \omega)\right]^{2} \\
& \times \int_{\mathbf{p}, \Omega} 2 A_{v} p^{-y_{v}} G_{v 0}(\mathbf{p}, \Omega) G_{v 0}(-\mathbf{p},-\Omega),
\end{aligned}
$$

where $\int_{\mathbf{p}, \Omega}=\int d^{d} \mathbf{p} /(2 \pi)^{d} \int_{-\infty}^{\infty} d \Omega / 2 \pi$ is an abbreviation for the integration over wave vector and frequency. The integration over wave vector can of course be further decomposed into an integration over angles and modulus. Equation (A1) can be used to obtain an expansion for the inverse propagator,

$$
\begin{aligned}
G_{u}^{-1}(\mathbf{k}, \omega)= & G_{u 0}^{-1}(\mathbf{k}, \omega)-\lambda \int_{\mathbf{p}, \Omega} 2 A_{v} p^{-y_{v}} G_{v 0}(\mathbf{p}, \Omega) \\
& \times G_{v 0}(-\mathbf{p},-\Omega)+O\left(\lambda^{2}\right) .
\end{aligned}
$$

Referring back to the structure of the bare propagator in (10), in order that the original model (1) be renormalizable in the hydrodynamic limit, the inverse effective propagator must have the form

$$
G_{u}^{-1}(\mathbf{k}, \omega)=\widetilde{\nu}+\widetilde{D}_{u} k^{2}-i \omega,
$$

where $\widetilde{\nu}$ and $\widetilde{D}_{u}$ are the effective, or renormalized, decay rate and diffusion constants for the $U$ field, respectively.

Since the one-loop correction integral in Eq. (A2) does not depend on either $\omega$ nor $k$, we can immediately conclude that the diffusion constant is unrenormalized at one-loop order, $\widetilde{D}_{u}=D_{u}$, while the decay rate renormalization is given by

$$
\widetilde{\nu}=\nu+\lambda \int_{\mathbf{p}, \Omega} 2 A_{v} p^{-y_{v}} G_{v 0}(\mathbf{p}, \Omega) G_{v 0}(-\mathbf{p},-\Omega) .
$$

The frequency integration over $\Omega$ can be performed exactly (e.g., by residues). Finally, eliminating a finite band of large wave numbers below the cutoff $\Lambda$ in the Wilsonian fashion then yields

$$
\widetilde{\nu}^{<}=\nu+\lambda A_{v} K_{d} \int_{\Lambda / s}^{\Lambda} d p \frac{p^{d-y_{v}-1}}{\left(\mu+D_{v} p^{2}\right)} .
$$

For an infinitesimally thin wave-number shell $(s=1+\delta, 0$ $<\delta \ll 1)$ we pass from an integral to a differential relation. After a further re-scaling according to (5) we obtain the first differential RG equation as displayed in (13). 


\section{APPENDIX B: VERTEX RENORMALIZATION}

Steps similar to those above are involved in the renormalization of the one-loop vertex (or, coupling $\lambda$ ). Transcribing the vertex diagram in the text back into algebraic quantities, we have that

$$
\begin{aligned}
\tilde{\lambda}= & \lambda-4 \lambda^{2} \int_{\mathbf{p}, \Omega} 2 A_{v} p^{-y_{v}} G_{v 0}(\mathbf{p}, \Omega) G_{v 0}(-\mathbf{p},-\Omega) \\
& \times G_{u 0}(-\mathbf{p},-\Omega),
\end{aligned}
$$

where we have set all external wave-numbers and frequencies to zero from the outset, in anticipation of the hydrodynamic limit. As before, the frequency integration can be per- formed immediately by means of residues. Eliminating a finite band of large wave-numbers yields

$$
\begin{aligned}
\tilde{\lambda}^{<}= & \lambda-4 \lambda^{2} A_{v} K_{d} \\
& \times \int_{\Lambda / s}^{\Lambda} d p \frac{p^{d-y_{v}-1}}{\left(\mu+D_{v} p^{2}\right)\left(\mu+\nu+\left[D_{u}+D_{v}\right] p^{2}\right)},
\end{aligned}
$$

For an infinitesimally thin wave-number shell $(s=1+\delta$, $0<\delta \ll 1)$ we pass from an integral to a differential relation. After a further rescaling according to Eq. (5) we obtain the last differential RG equation as displayed in Eq. (13).
[1] For a recent and extensive review, see Daniel Walgraef, Spatio-Temporal Pattern Formation (Springer, New York, 1997).

[2] M. C. Cross and P. C. Hohenberg, Rev. Mod. Phys. 65(3), 851 (1993).

[3] B. P. Lee, J. Phys. A 27, 2633 (1984), B. P. Lee and J. Cardy, Phys. Rev. E 50, 3287 (1994); J. Stat. Phys. 80, 971 (1995).

[4] P. Gray and S. K. Scott, Chem. Eng. Sci. 38, 29 (1983); 39, 1087 (1984); J. Chem. Phys. 89, 22 (1985).

[5] J. E. Pearson, Science 261, 189 (1993).

[6] F. Lesmes, D. Hochberg, F. Morán, and J. Pérez-Mercader, Phys. Rev. Lett. 91, 238301 (2003).

[7] M. Doi, J. Phys. A 9, 1465 (1976); 9, 1479 (1976).

[8] L. Peliti, J. Phys. (Paris) 46, 1469 (1985).

[9] P. Grassberger and M. Scheunert, Fortschr. Phys. 28, 547 (1980); P. Grassberger, F. Krause, and T. von der Twer, J. Phys. A 17, L105 (1984).
[10] M. J. Howard and U. C. Täuber, J. Phys. A 30, 7721 (1997).

[11] E. Frey and U. C. Täuber, Phys. Rev. E 50, 1024 (1994).

[12] P. C. Hohenberg and B. I. Halperin, Rev. Mod. Phys. 49, 435 (1977).

[13] S. K. Ma, Modern, Theory of Critical Phenomena (Benjamin/ Cummings Publishing Company, Reading, MA, 1976).

[14] A.-L. Barabási and H. E. Stanley, Fractal Concepts in Surface Growth (Cambridge University Press, Cambridge, UK, 1995).

[15] D. Hochberg, C. Molina-París, J. Pérez-Mercader, and M. Visser, Phys. Rev. E 60, 6343 (1999).

[16] The required integrals can be found in I. S. Gradsteyn and I. M. Ryzik, Tables of Integrals, Series, and Products (Academic Press, New York, 1980).

[17] J. Pérez-Mercader, in Astrobiology: The Quest for the Conditions of Life, edited by G. Hormeck and C. Baumstark-Khan (Springer-Verlag, New York, 2001). 